\title{
Functional Redesign of Mantis 2.0, a Hybrid Leg-Wheel Robot for Surveillance and Inspection
}

\author{
Luca Bruzzone • Pietro Fanghella
}

Received: 17 December 2014 / Accepted: 6 May 2015 / Published online: 12 June 2015

(C) Springer Science+Business Media Dordrecht 2015

\begin{abstract}
The paper discusses the redesign of the second version of the Mantis hybrid leg-wheel mobile robot, conceived for surveillance and inspection tasks in unstructured indoor and outdoor environments. This small-scale ground mobile robot is characterized by a main body equipped with two front actuated wheels, a passive rear axle and two rotating legs. Motion on flat and even ground is purely wheeled in order to obtain high speed, high energetic efficiency and stable camera vision; only in case of obstacles or ground irregularities the front legs realize a mixed leg-wheel locomotion to increase the robot climbing ability; in particular, the outer profile of the legs, inspired by the praying mantis, is specially designed to climb square steps. The multibody simulations and the experimental tests on the first prototype have shown the effectiveness of the mixed leg-wheel locomotion not only for step climbing, but also on uneven and yielding terrains. Nevertheless, extensive experimental tests have shown that the front wheels may slip in the last phase of step climbing in case of contact with some materials. In order to overcome this problem, the leg design has been modified with the introduction of auxiliary passive wheels, which reduce friction between legs and step upper surface; these wheels are connected to the legs by one-way bearings, in order to rotate only
\end{abstract}

L. Bruzzone $(\bowtie) \cdot$ P. Fanghella

DIME Department, University of Genova, Genova, Italy

e-mail: bruzzone@dimec.unige.it when they are pulled by the front wheels, and remaining locked when they have to push forward the robot. The influence of the auxiliary wheels on the front wheels slippage is investigated by means of theoretical analysis and multibody simulations.

Keywords Mobile robot - Hybrid leg-wheel locomotion $\cdot$ Stability analysis $\cdot$ Multibody simulation

\section{Introduction}

In the last years, the scientific and industrial research interest about ground mobile robots is continuously growing. Some of the main application fields are defense and homeland security [1, 2], surveillance [3], exploration and rescue in dangerous locations [4-6]. In the research and industrial scenarios there is a wide variety of proposed locomotion systems, and it is not easy to perform a complete and synthetic classification and comparison; if we exclude robots with locomotion principles oriented to specialpurpose applications (such as snake-like, slithering, adhesive or jumping robots), it is possible to identify three main categories of ground mobile robots: wheeled (W), tracked (T), and legged (L); moreover, four hybrid categories can be derived by combination (legs-wheels, LW, legs-tracks, LT, wheels-tracks, WT, legs-wheels-tracks, LWT). 
The pros and cons of these seven categories of locomotion systems are outlined in [7-9]. Usually, locomotion systems including wheels (W, LW, WT, LWT) are characterized by high maximum speed and energetic efficiency; locomotion system including tracks (T, WT, LT, LWT) are suitable for uneven and soft terrains; locomotion systems including legs (L, LW, LT, LWT) are characterized by high obstacle climbing ability, but also by high mechanical and control complexity.

In order to maximize the operative flexibility of ground mobile robots, the main difficulty is to design a locomotion system which fulfils conflicting requirements, such as high speed and efficiency on flat terrains and obstacle climbing ability. This problem is relevant especially for medium-scale and large-scale robots, because the inertial forces during obstacle climbing are not negligible. On the other hand, if we consider small-scale robots, the inertial forces related to trajectory discontinuities are usually not critical, and this allows to adopt simpler locomotion mechanisms; therefore there is a wide variety of locomotion architectures with simplified mechanics, which are not suitable for heavy robots but that provide an excellent compromise between performance and cost for lightweight robots. Examples of this design approach for small-scale robots are RHex [10], Whegs [11] and Loper [12]; these robots are characterized by a locomotion principle based on one-degree-of-freedom rotating legs which perform differential steering; their mechanical design and their control are quite simple; nevertheless, thanks to the design of their flexible legs, their speed and obstacle climbing ability are rather good. On the other hand, their locomotion is highly oscillating, and this is a great drawback for vision-based applications.

Another example of this design approach for smallscale robots is the stepping triple wheel: this hybrid LW locomotion system is characterized by three legs placed at $120^{\circ}$, with a wheel at each leg end $[3,13-$ 15]. Locomotion is wheeled on flat terrains, while the legs rotate with respect to the main body to climb obstacles and unevenness. Unlike rotating legs robots, in the wheeled locomotion stepping triple wheel robots have high energetic efficiency and steady vision, without camera oscillations.

The rest of this paper deals with the Mantis architecture [16-18], which is an alternative to rotating legs and stepping triple wheels concepts. A multibody model of the robot performing step climbing has been validated through a comparison with experimental results [16], and this model has been used to analyse the influence of the main geometric parameters and of the leg-wheel motion planning on the stepclimbing process [17]. In [18] the prototype behaviour in different situations, such as locomotion on uneven terrains, is discussed. The experimental campaign has shown that for some types of step material and climbing conditions, the friction between front wheels and ground is weaker than friction between the leg ends and ground, and this may cause the front wheel slippage and, consequently, the robot stop in the last phase of step climbing. To avoid this effect, the legs have been modified with the introduction of auxiliary passive wheels, to switch from sliding friction to a lower rolling resistance between legs and step upper surface; the auxiliary wheels are connected to the legs by oneway bearings, in order to rotate only when they are pulled by the front wheels, remaining locked when they have to generate traction. In the present paper, the influence of the auxiliary wheels on the front wheels slippage and robot motion is analysed by means of theoretical analysis and multibody simulations.

\section{The Mantis Architecture}

The Mantis robot has been conceived as a generalpurpose small scale platform (about $350 \times 300 \times 200$ $\mathrm{mm}$ ) for security and inspection applications which require a small surveillance equipment (maximum payload mass: $1 \mathrm{~kg}$ ). It has been designed to obtain a great operative flexibility in structured and unstructured environments, going up and down stairs with standard-size steps (160 mm of rise, $320 \mathrm{~mm}$ of going, according to UNI 10804:1999) and pivoting around a vertical axis for maximum manoeuvrability in narrow spaces; other considered specifications are steady locomotion on flat grounds, to obtain high-quality camera vision, but also the capability of moving in outdoor environments with uneven terrains and irregular obstacles.

Starting from these general requirements, the basic idea of Mantis is to adopt pure wheeled locomotion on flat grounds, without camera oscillations, while the rotating legs provide additional traction only in case of steps, stairs, other obstacles or terrain unevenness. 

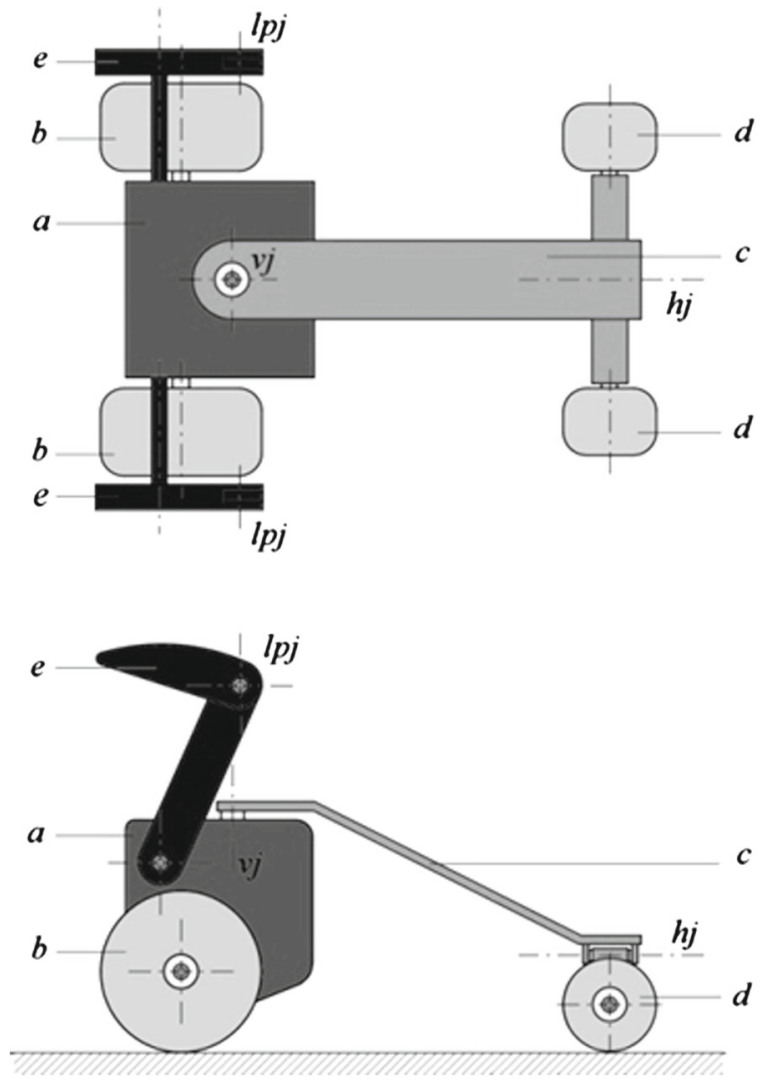

Fig. 1 The Mantis robot

The robot (Fig. 1) is characterized by a main body (a) and two actuated front wheels $(b)$, which perform differential steering. The passive rear axle $(c)$, which carries two idle wheels $(d)$, grants the robot stability. The revolute joint $v j$ permits a relative motion between $a$ and $c$ while steering, and the revolute joint $h j$ permits the rear axle roll in case of unevenness. Also the two rotating legs $(e)$ are independently actuated, so in case of hybrid leg-wheel locomotion on uneven terrains, when traction is generated concurrently by legs and wheels, it is possible to steer imposing higher speed to the front wheel and to the leg on the outside of the turn; their praying mantis shape has been conceived to climb square steps, by grasping the step upper surface and lifting up the robot body (Fig. 2, left). Each leg is composed of two links in relative motion with a passive degree of freedom: the leg ends can bend thanks to the revolute joints $l p j$, and elastic elements are added to soften impacts during the step descent (Fig. 2, right). The passive degree of freedom of the legs is unidirectional: the leg ends can bend only internally, so they can move for shock absorption, while they are locked by the elastic preload during climbing.

The first experimental tests on the Mantis 1.0 prototype, performed with manual radio-control by a human operator, have shown that the combined use of legs and wheels with differential steering is very efficient on irregular terrains (Fig. 3) and provides excellent step-climbing ability (Fig. 4).

Mantis performs step climbing as shown in Fig. 5:

- while the robot approaches the step by wheeled locomotion, the legs start to rotate forward, grasping the step and lifting up the robot body (frames $1-3)$;

- in the position of frame 4, the legs stop; due to the gravity force, the robot rotates around the contact point between legs and step upper surface;

- when the front wheels touch the step upper surface (frame 5), the legs rotate backward for a small angle, in order to lift up the rear axle (frame 6);

- the front wheels continue to rotate pulling forward the robot (frames 6,7); when the rear axle is above
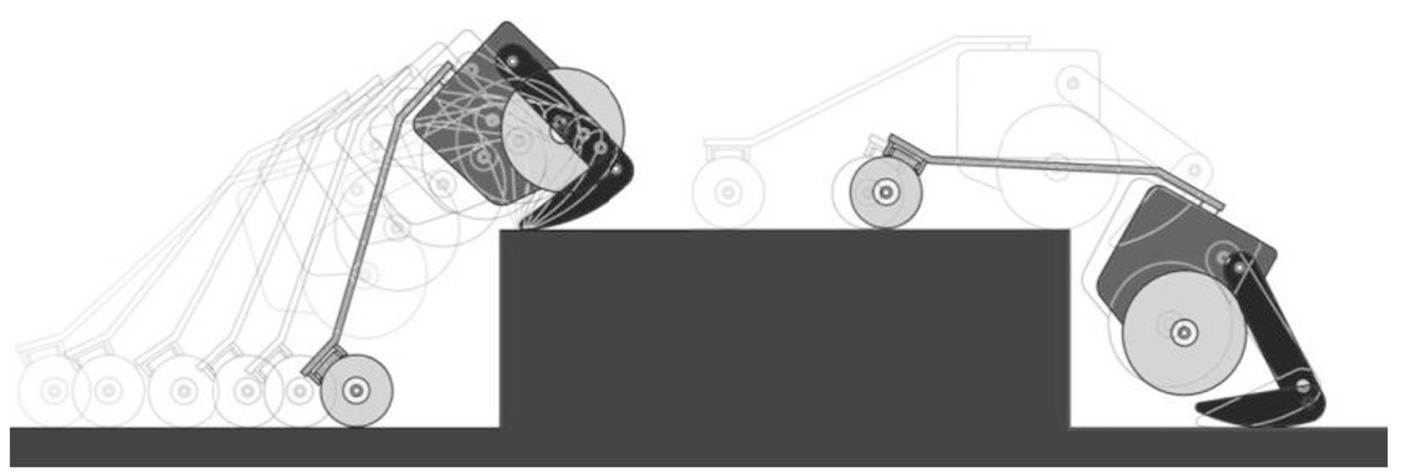

Fig. 2 Step ascent and descent 


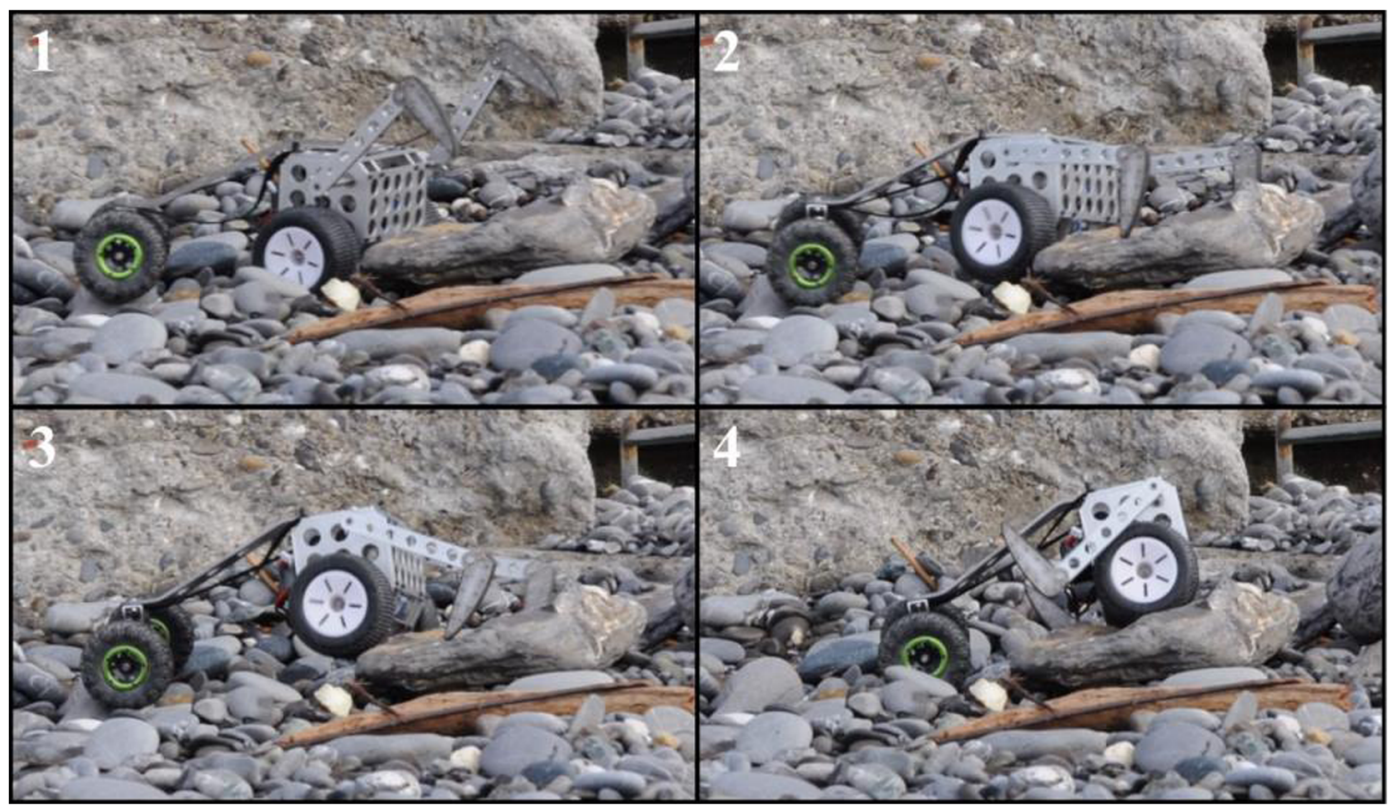

Fig. 3 Locomotion on uneven terrain

the step (frame 7), the legs start to rotate frontward lowering the rear axle until it touches the step; then the legs lose contact with the ground, and locomotion becomes again purely wheeled (frame 8).

In the final phase of the step climbing sequence, after that the rear axle is elevated above the step (frames

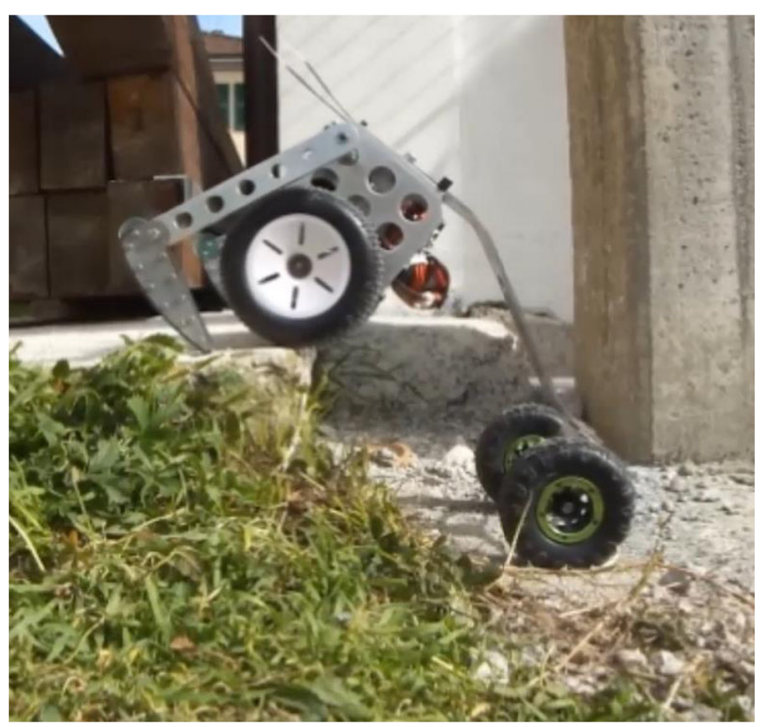

Fig. 4 Step climbing
6-7), the forward motion is originated by the friction force between front wheels and step upper surface, and hindered by the friction force between legs and step upper surface. Usually the first friction force overcomes the second, because the friction coefficient between front wheels and step is higher than the one between legs and step. Nevertheless, the experimental test campaign has shown that in presence of certain types of step surfaces and climbing conditions the front wheels may slip, lowering the reliability of the step climbing manoeuvre. In Sections 3 to 5 stability and no-slip condition during step-climbing are discussed.

\section{Geometrical Model}

Table 1 collects the main robot geometrical parameters, shown in Fig. 6; this figure shows also the variable leg angle $\theta_{l}$, which is null when the leg is vertical. Step climbing can be analysed considering a planar model: the robot reference frame $\left(x^{\prime}, z^{\prime}\right)$ has its center on the front wheels axis $O_{f}$, and the point $O_{l}$ is the intersection of the axis of the actuated leg revolute joints with the $x^{\prime} z^{\prime}$ plane.

For sake of generality, a nondimensional approach is used, based on the geometrical ratios collected in 
Fig. 5 Step climbing

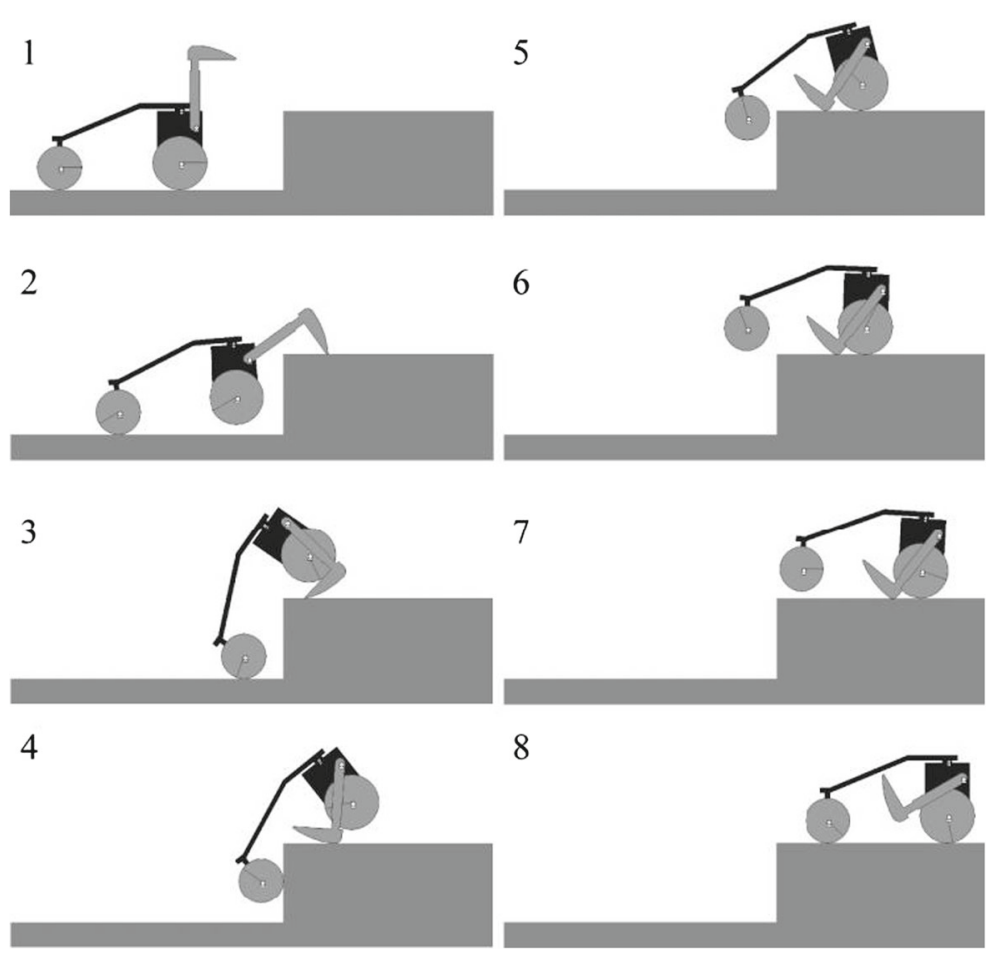

Table 2. The third column of this table shows the corresponding values of the Mantis 1.0 prototype. Let us note that only $\alpha$ is influenced by the step height, which is obviously variable; the value of $\alpha$ of Table 2 is related to the standard rise $(h=160 \mathrm{~mm})$.

The maximum pitch angle position during step climbing is shown in Fig. 7: $O_{l}$ is aligned vertically with the contact point between leg and step upper surface. Since the leg outer profile is a circular arc with radius $r_{l}$ and center $O_{l}$, there is a range of leg positions which corresponds to the maximum pitch angle. In these positions, the robot weight is not sufficient to overcome the elastic preload of the passive degrees of

Table 1 Main Geometrical Parameters

\begin{tabular}{ll}
\hline Parameter & Symbol \\
\hline front wheel radius & $r_{f}$ \\
rear wheel radius & $r_{r}$ \\
leg radius & $r_{l}$ \\
$x$-coordinate of $O_{l}$ in the robot reference frame & $x_{l}^{\prime}$ \\
$z$-coordinate of $O_{l}$ in the robot reference frame & $z_{l}^{\prime}$ \\
wheelbase & $i$ \\
step height & $h$ \\
\hline
\end{tabular}

freedom of the legs; therefore the maximum pitch can be obtained by the geometrical model of Fig. 7:

$$
\begin{aligned}
\theta_{\max }= & \varphi_{\max }-\delta=\arcsin \left(\frac{\gamma_{l}+\frac{2}{\alpha}-\beta}{\sqrt{\left(\gamma+\xi_{l}\right)^{2}+\left(\psi_{l}+1-\beta\right)^{2}}}\right) \\
& -\arctan \left(\frac{\psi_{l}+1-\beta}{\gamma+\xi_{l}}\right)
\end{aligned}
$$

\section{Stability Analysis During Step Climbing}

The static stability condition is verified if the vertical projection of the center of mass is within the support polygon [19]. With reference to the maximum pitch angle position shown in Fig. 7, the static stability condition is verified if:

$\delta_{G}+\theta_{\max }=\arctan \left(\frac{\psi_{G}+1-\beta}{\gamma+\xi_{G}}\right)+\theta_{\max }<\frac{\pi}{2}$

where $\xi_{G}=x_{G}^{\prime} / r_{f}$ and $\psi_{G}=z_{G}^{\prime} / r_{f}$ represent the normalized coordinates of the robot overall center of mass $G$ in the robot reference frame with the legs in rest position, pointing backward $\left(\theta_{l}=90^{\circ}\right)$. In the stability analysis these coordinates are considered 
Fig. 6 Main geometrical parameters

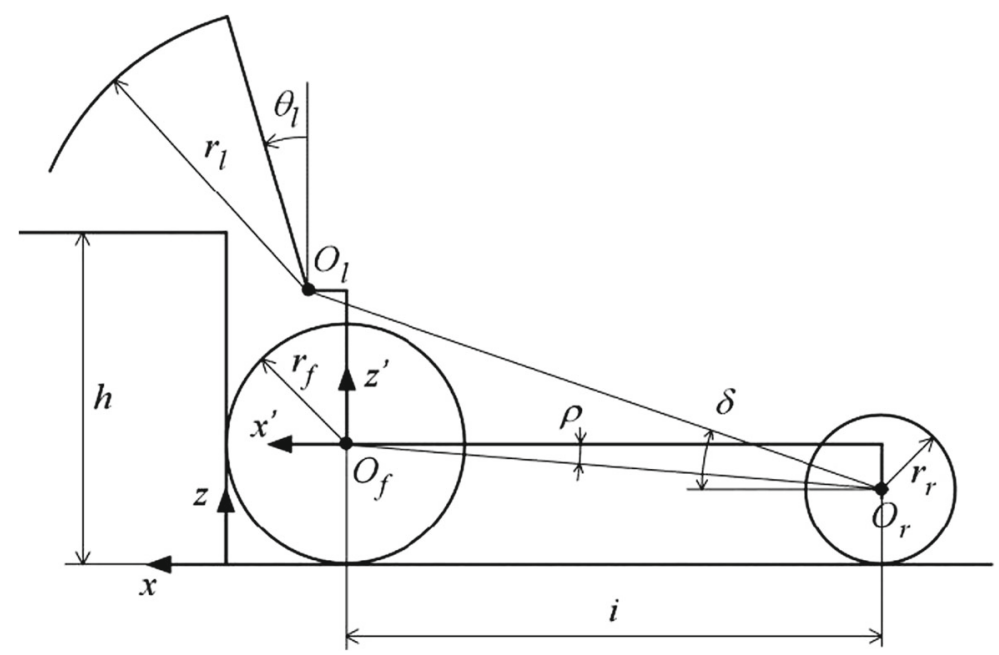

independent of the leg position, and this hypothesis is conservative, because moving the legs in step climbing improves stability.

Given the center of mass position, considering Eqs. 1 and 2, it is possible to obtain the limit of stability condition as a function of the robot design parameters and of the step height, which is represented by the following equation:

$$
\begin{aligned}
& \arctan \left(\frac{z_{G}^{\prime}+r_{f}-r_{r}}{i+x_{G}^{\prime}}\right)+\arcsin \left(\frac{r_{l}+h-r_{r}}{\sqrt{\left(i+x_{l}^{\prime}\right)^{2}+\left(z_{l}^{\prime}+r_{f}-r_{r}\right)^{2}}}\right) \\
&-\arctan \left(\frac{z_{l}^{\prime}+r_{f}-r_{r}}{i+x_{l}^{\prime}}\right) \\
&=\arctan \left(\frac{\psi_{G}+1-\beta}{\gamma+\xi_{G}}\right)+\arcsin \left(\frac{\gamma_{l}+\frac{2}{\alpha}-\beta}{\sqrt{\left(\gamma+\xi_{l}\right)^{2}+\left(\psi_{l}+1-\beta\right)^{2}}}\right) \\
&-\arctan \left(\frac{\psi_{l}+1-\beta}{\gamma+\xi_{l}}\right)=\frac{\pi}{2}
\end{aligned}
$$

Table 2 Geometrical Ratios

\begin{tabular}{lll}
\hline Ratio symbol & Definition & Prototype value \\
\hline$\alpha$ & $2 r_{f} / h$ & 0.69 \\
$\beta$ & $r_{r} / r_{f}$ & 0.82 \\
$\gamma$ & $i / r_{f}$ & 4.35 \\
$\xi_{l}$ & $x_{l}^{\prime} / r_{f}$ & 0.58 \\
$\psi_{l}$ & $z_{l}^{\prime} / r_{f}$ & 1.45 \\
$\gamma_{l}$ & $r_{l} / r_{f}$ & 2.90 \\
\hline
\end{tabular}

Starting from Eq. 3 it is possible to obtain the expression of $(1 / \alpha)_{\max }$, which represents the normalized height of the maximum climbable step:

$$
\begin{aligned}
\left(\frac{1}{\alpha}\right)_{\max }= & \frac{\beta-\gamma_{l}}{2}+\frac{1}{2} \cos \left(\arctan \left(\frac{\beta-\psi_{G}-1}{\gamma+\xi_{G}}\right)\right. \\
& \left.-\arctan \left(\frac{\beta-\psi_{l}-1}{\gamma+\xi_{l}}\right)\right) \cdot \sqrt{\left(\gamma+\xi_{l}\right)^{2}+\left(\psi_{l}+1-\beta\right)^{2}}
\end{aligned}
$$

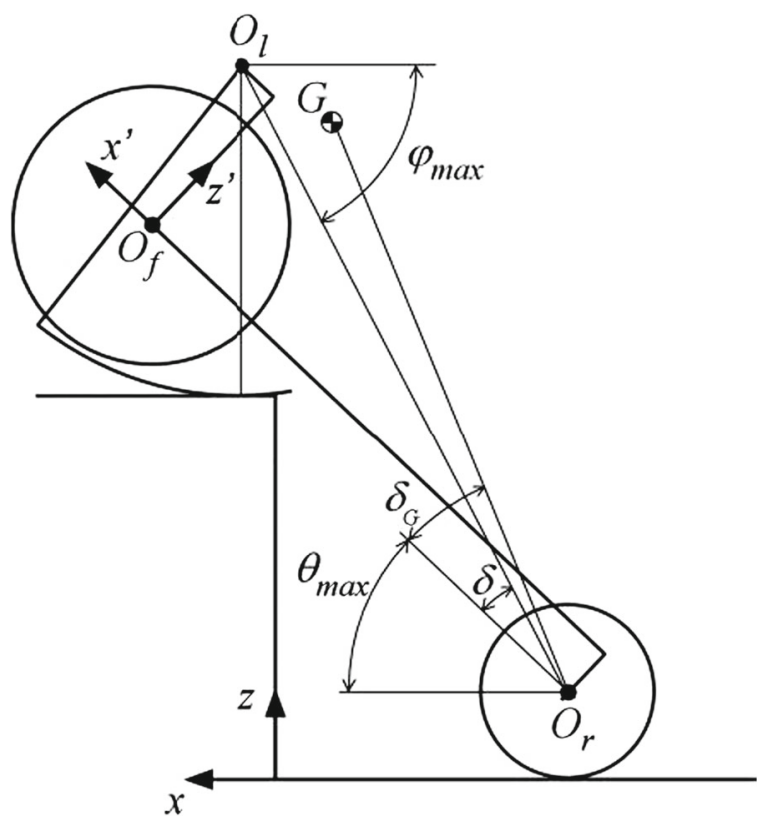

Fig. 7 Maximum pitch angle position during step climbing 
The ratio $(1 / \alpha)_{\max }$ corresponds to the maximum step height $h_{\max }$ :

$$
\begin{aligned}
h_{\max }= & \left(r_{r}-r_{l}\right)+\cos \left(\arctan \left(\frac{r_{r}-z_{G}^{\prime}-r_{f}}{i+x_{G}^{\prime}}\right)\right. \\
& \left.-\arctan \left(\frac{r_{r}-z_{l}^{\prime}-r_{f}}{i+x_{l}^{\prime}}\right)\right) \cdot \sqrt{\left(i+x_{l}^{\prime}\right)^{2}+\left(z_{l}^{\prime}+r_{f}-r_{r}\right)^{2}}
\end{aligned}
$$

The value of $(1 / \alpha)_{\max }$ is 1.55 for the configuration of Table 2. Eqs. 4 and 5 define the influence of the wheelbase and of the leg radius on stability in the maximum pitch angle position; nevertheless these parameters are bounded by other functional requirements, not related to step climbing:

- if the wheelbase is too long, the robot manoeuvrability in narrow spaces becomes poor, because the robot cannot pivot around a vertical axis by imposing equal and opposite front wheel speeds;

- the legs have to be sufficiently long to touch the ground, in order to perform a mixed leg-wheel locomotion on uneven terrains, and this condition is represented non-dimensionally by the inequality $\gamma_{l}>\psi_{l}+1$.

In the last phase of the step-climbing manoeuvre, when the robot is suspended on front wheels and legs (Fig. 5, frames 5 to 7), the stability condition is verified if the center of mass vertical projection lies between the contact points of front wheels and legs

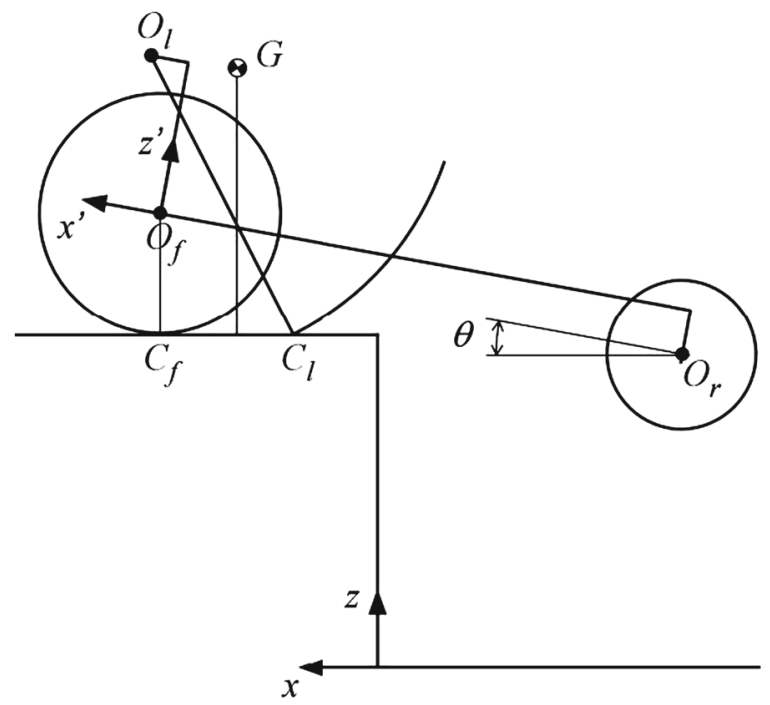

Fig. 8 Final position of the step climbing without auxiliary wheels with the step upper surface $C_{f}$ and $C_{l}$, (Fig. 8), that is:

$x_{C_{l}}<x_{G}<x_{C_{f}}$

Considering the geometry shown in Fig. 8 it is possible to express the inequalities (6) as follows:

$$
\begin{aligned}
0 & <\psi_{G} \sin \theta-\xi_{G} \cos \theta<\psi_{l} \sin \theta-\xi_{l} \cos \theta \\
& +\sqrt{\gamma_{l}^{2}-\left(1+\psi_{l} \cos \theta+\xi_{l} \sin \theta\right)^{2}}
\end{aligned}
$$

Fortunately, all the heaviest components of the robot (gearmotors, gear pairs, front wheels, legs, front wheel shafts with bearings, leg shafts with bearings, batteries, control devices) are placed on the main body, with centres of mass close to $O_{f}$; also the payload (camera, environmental sensors) is located on the upper/front part of the main body; on the other hand, the rear axle supports only the rear idle wheels, much lighter than the front ones; therefore, the stability condition expressed by the inequalities (6) is easily fulfilled. A shorter wheelbase $i$ is favourable for the stability condition (6) and for the robot manoeuvrability in narrow spaces, but the minimum value of $i$ is bounded by the maximum pitch angle stability condition (2).

As discussed in Section 2, in the last phase of the step climbing manoeuvre (Fig. 5, frames 6-7) front wheels slippage may occur for particular friction characteristics of the step horizontal surface; to solve this problem, the proposed solution is to introduce auxiliary wheels connected to the legs by one-way bearings, in order to have rolling friction instead of sliding

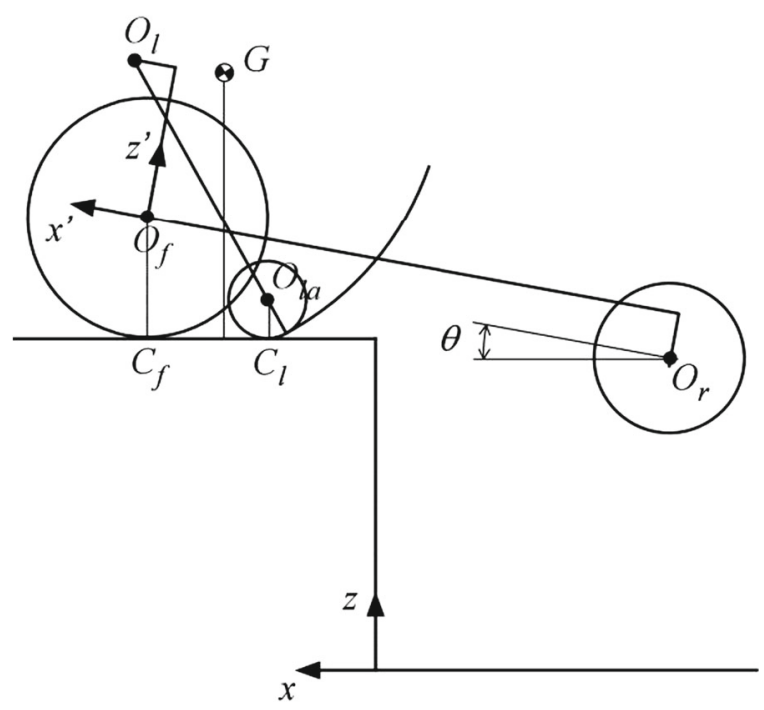

Fig. 9 Final position of the step climbing with auxiliary wheels 
friction between legs and step in this phase; one-way bearings are necessary to avoid backward rolling when the robot is rotating around the contact point between legs and step (Fig. 5, frames 4-5) and in other operative conditions, for example during mixed leg-wheel locomotion on uneven terrains. The auxiliary wheels have radius $r_{l a}$ and their axes are placed in $O_{l a}$ at a distance $\left(r_{l}-r_{l a}\right)$ form $O_{l}$, as shown in Fig. 9.

Auxiliary wheels do not influence stability in the maximum pitch position of Fig. 7, but they influence stability in the final position of step climbing. Introducing the geometrical ratio $\gamma_{l a}=r_{l a} / r_{f}$ and considering the geometry shown in Fig. 9 it is possible to express the stability condition (6) as follows:

$\begin{aligned} 0< & \psi_{G} \sin \theta-\xi_{G} \cos \theta<\psi_{l} \sin \theta-\xi_{l} \cos \theta \\ & +\sqrt{\left(\gamma_{l}-\gamma_{l a}\right)^{2}-\left(1-\gamma_{l a}+\psi_{l} \cos \theta+\xi_{l} \sin \theta\right)^{2}}\end{aligned}$

\section{No-Slip Condition During Step Climbing}

When the robot is suspended on front wheels and legs in static conditions, without auxiliary wheels (Fig. 8), it is possible to evaluate the normal reaction forces
$R_{f}$ and $R_{l}$ (in $C_{f}$ and $C_{l}$ ) by imposing vertical and rotational equilibrium conditions ( $m$ is the overall robot mass):

$R_{f}=m g\left(1-\frac{\psi_{G} \sin \theta-\xi_{G} \cos \theta}{\psi_{l} \sin \theta-\xi_{l} \cos \theta+\sqrt{\gamma_{l}^{2}-\left(1+\psi_{l} \cos \theta+\xi_{l} \sin \theta\right)^{2}}}\right)$

$R_{l}=m g \frac{\psi_{G} \sin \theta-\xi_{G} \cos \theta}{\psi_{l} \sin \theta-\xi_{l} \cos \theta+\sqrt{\gamma_{l}^{2}-\left(1+\psi_{l} \cos \theta+\xi_{l} \sin \theta\right)^{2}}}$

The no-slip condition is:

$R_{f} f_{f}>R_{l} f_{l}$

where $f_{f}$ is the static friction coefficient between wheels and step upper surface and $f_{l}$ is the static friction coefficient between legs end and step upper surface; using Eqs. 9 and 10 the no-slip condition (11) can be expressed by the following inequality:

$$
\frac{\psi_{l} \sin \theta-\xi_{l} \cos \theta+\sqrt{\gamma_{l}^{2}-\left(1+\psi_{l} \cos \theta+\xi_{l} \sin \theta\right)^{2}}}{\psi_{G} \sin \theta-\xi_{G} \cos \theta}>1+\frac{f_{l}}{f_{f}}
$$

When the auxiliary wheels are present, the normal reaction forces $R_{f}$ and $R_{l}$ can be evaluated similarly considering the geometry of Fig. 9:

$R_{f}=m g\left(1-\frac{\psi_{G} \sin \theta-\xi_{G} \cos \theta}{\psi_{l} \sin \theta-\xi_{l} \cos \theta+\sqrt{\left(\gamma_{l}-\gamma_{l a}\right)^{2}-\left(1-\gamma_{l a}+\psi_{l} \cos \theta+\xi_{l} \sin \theta\right)^{2}}}\right)$,

$$
R_{l}=m g \frac{\psi_{G} \sin \theta-\xi_{G} \cos \theta}{\psi_{l} \sin \theta-\xi_{l} \cos \theta+\sqrt{\left(\gamma_{l}-\gamma_{l a}\right)^{2}-\left(1-\gamma_{l a}+\psi_{l} \cos \theta+\xi_{l} \sin \theta\right)^{2}}} .
$$

In this case the no-slip condition is:

$R_{f} f_{f}>R_{l} f_{l r}$, where $f_{l r}$ is the rolling resistance coefficient between auxiliary wheels and step upper surface; using Eqs. 13 and 14 the no-slip condition (15) can be expressed by the following inequality:

$\frac{\psi_{l} \sin \theta-\xi_{l} \cos \theta+\sqrt{\left(\gamma_{l}-\gamma_{l a}\right)^{2}-\left(1-\gamma_{l a}+\psi_{l} \cos \theta+\xi_{l} \sin \theta\right)^{2}}}{\psi_{G} \sin \theta-\xi_{G} \cos \theta}>1+\frac{f_{l r}}{f_{f}}$. 
The comparison between Eqs. 12 and 16 shows the positive influence of the auxiliary wheels during the critical final phase of step climbing (Fig. 5, frames 6,7$)$, which is performed with slightly negative pitch angles $\left(\theta \approx 5^{\circ}\right)$ to avoid contact between step edge and rear wheels: as a matter of fact, the numerators of the left sides of Eqs. 12 and 16 are very close, since $\gamma_{l a}<<\gamma_{l}$, while the rolling resistance coefficient $f_{l r}$ is much lower than $f_{l}$; therefore, it is much easier to fulfil (16) than (12) for any ground condition.

\section{Dynamic Simulations}

The effectiveness of the introduction of the legs auxiliary wheels in the step climbing process has been assessed by multibody simulation, comparing the two multibody models (without and with auxiliary wheels) shown respectively in Fig. 10a and b.

The first model is composed of four bodies: $\mathrm{B}_{1}$, which represents the main body $a$ and the rear axle $c$; $B_{2}$, which represents the front wheels, is connected to $B_{1}$ by the revolute joint $R_{1-2} ; B_{3}$, which represents the rear wheels, is connected to $\mathrm{B}_{1}$ by the revolute joint $\mathrm{R}_{1-3} ; \mathrm{B}_{4}$, which represents the legs, is connected to $B_{1}$ by the revolute joint $R_{1-4}$. In the second model body $\mathrm{B}_{5}$ represents the auxiliary wheels and is connected to $\mathrm{B}_{4}$ by the revolute joint $\mathrm{R}_{4-5}$, which is locked in one rotation direction in order to model the one-way bearings behaviour.

In both models, the motions of the revolute joints $\mathrm{R}_{1-2}$ and $\mathrm{R}_{1-4}$ are determinate, because their positions are imposed by the actuators as functions of time. The contact force $\mathrm{C}_{\mathrm{i}-\mathrm{G}}(i=2 \ldots 4$ for both models, and also $i=5$ for the second model) is defined between the body $\mathrm{B}_{i}$ and the ground.

Simulations have been performed using the Mantis 1.0 geometry, defined in Table 2, with a wheel radius $r_{f}=55 \mathrm{~mm}$ and considering the $160 \mathrm{~mm}$ standard rise; for the second model the radius of the auxiliary wheels is $15 \mathrm{~mm}$.

The masses of the bodies 1 to 4 have been derived from the first prototype: $m_{\mathrm{B} 1}=1.9 \mathrm{~kg} ; m_{\mathrm{B} 2}=$ $0.5 \mathrm{~kg} ; m_{\mathrm{B} 3}=0.2 \mathrm{~kg} ; m_{\mathrm{B} 4}=0.4 \mathrm{~kg}$; the mass of $\mathrm{B}_{5}$ is $0.04 \mathrm{~kg}$. The definition of the unilateral contact forces $\mathrm{C}_{\mathrm{i}-\mathrm{G}}$ is based on the real compliance and friction coefficients of tires and legs tips, which have been measured on the prototype.
The step climbing manoeuvre starts (Fig 5, frame 1) with vertical legs and initial distance $d_{f 0}$ of the front wheel axis $O_{f}$ from the step; during the whole manoeuvre the front wheels speed $\omega_{w}$ is constant, while the motion law of the legs is more complex. As a matter of fact, the legs rotate frontward to grasp the step and lift the robot body (Fig. 5, frames 2, 3), then the legs stop (Fig. 5, frame 4); while legs are stopped, the robot starts to rotate around the contact point $P$ between legs and step upper surface thanks to the gravity force, because its center of mass is placed forward with respect to $P$. After that the front wheels touch the step upper surface (Fig. 5, frame 5) the legs rotate backward for a small angle, in order to lift up the rear axle (Fig. 4, frame 6); the front wheels continue to rotate pulling forward the robot (Fig. 5, frame 7); when the rear axle is above the step, the legs start to rotate frontward and lose contact with the ground (Fig. 5, frame 8).

Accordingly, the motion law of the legs is divided in the five phases $A$ to $E$, shown in Fig. 11:

A. (Fig. 5, frames 1-4): forward rotation with constant acceleration $\alpha_{\mathrm{A}}$ for $t \in\left[0, t_{\mathrm{A} 1}\right)$; constant speed $\omega_{\mathrm{A}}$ for $t \in\left[t_{\mathrm{A} 1}, t_{\mathrm{A} 2}\right)$; constant deceleration $-\alpha_{\mathrm{A}}$ for $t \in\left[t_{\mathrm{A} 2}, t_{\mathrm{B}}\right)$;

B. (Fig. 5, frames 4-5): stop at angle $\theta_{\mathrm{B}}$ for $t \in\left[t_{\mathrm{B}}\right.$, $\left.t_{\mathrm{C} 0}\right)$

C. (Fig. 5, frames 5-6): backward rotation with constant acceleration $-\alpha_{\mathrm{C}}$ for $t \in\left[t_{\mathrm{C} 0}, t_{\mathrm{C} 1}\right)$, constant speed $-\omega_{\mathrm{C}}$ for $t \in\left[t_{\mathrm{C} 1}, t_{\mathrm{C} 2}\right)$, constant deceleration $\alpha_{\mathrm{C}}$ for $t \in\left[t_{\mathrm{C} 2}, t_{\mathrm{D}}\right)$;

D. (Fig. 5, frames 6-7): stop at angle $\theta_{\mathrm{D}}$ for $t \in\left[t_{\mathrm{D}}\right.$, $\left.t_{\mathrm{E} 0}\right)$

E. (Fig. 5, frames 7-8): forward rotation with constant acceleration $\alpha_{\mathrm{E}}$ for $t \in\left[t_{\mathrm{E} 0}, t_{\mathrm{E} 1}\right)$ and then with constant speed $\omega_{\mathrm{E}}$ for $t \geq t_{\mathrm{E} 1}$, to reach the leg rest position.

The comparison of the two multibody models of Fig. 10 confirms the effectiveness of the introduction of the auxiliary wheels. Figures 12 to 15 show the simulation results of both models with the motion law defined by the following parameters:

- initial distance $d_{f 0}=210 \mathrm{~mm}$

- front wheels: $\omega_{w}=150^{\circ} / \mathrm{s}$

- legs: $t_{\mathrm{A} 1}=0.1 \mathrm{~s} ; t_{\mathrm{A} 2}=3.8 \mathrm{~s} ; t_{\mathrm{B}}=3.9 \mathrm{~s} ; t_{\mathrm{C} 0}=$ $4.10 \mathrm{~s} ; t_{\mathrm{C} 1}=4.20 \mathrm{~s} ; t_{\mathrm{C} 2}=4.30 \mathrm{~s} ; t_{\mathrm{D}}=4.40 \mathrm{~s}$; $t_{\mathrm{E} 0}=4.90 \mathrm{~s} ; t_{\mathrm{E} 1}=5.00 \mathrm{~s} ; \alpha_{\mathrm{A}}=\alpha_{\mathrm{C}}=\alpha_{\mathrm{E}}=$ $600^{\circ} / \mathrm{s}^{2} ; \omega_{\mathrm{A}}=\omega_{\mathrm{C}}=\omega_{\mathrm{E}}=60^{\circ} / \mathrm{s}$ 
Fig. 10 Planar multibody model without auxiliary wheels (a) and with auxiliary wheels (b)

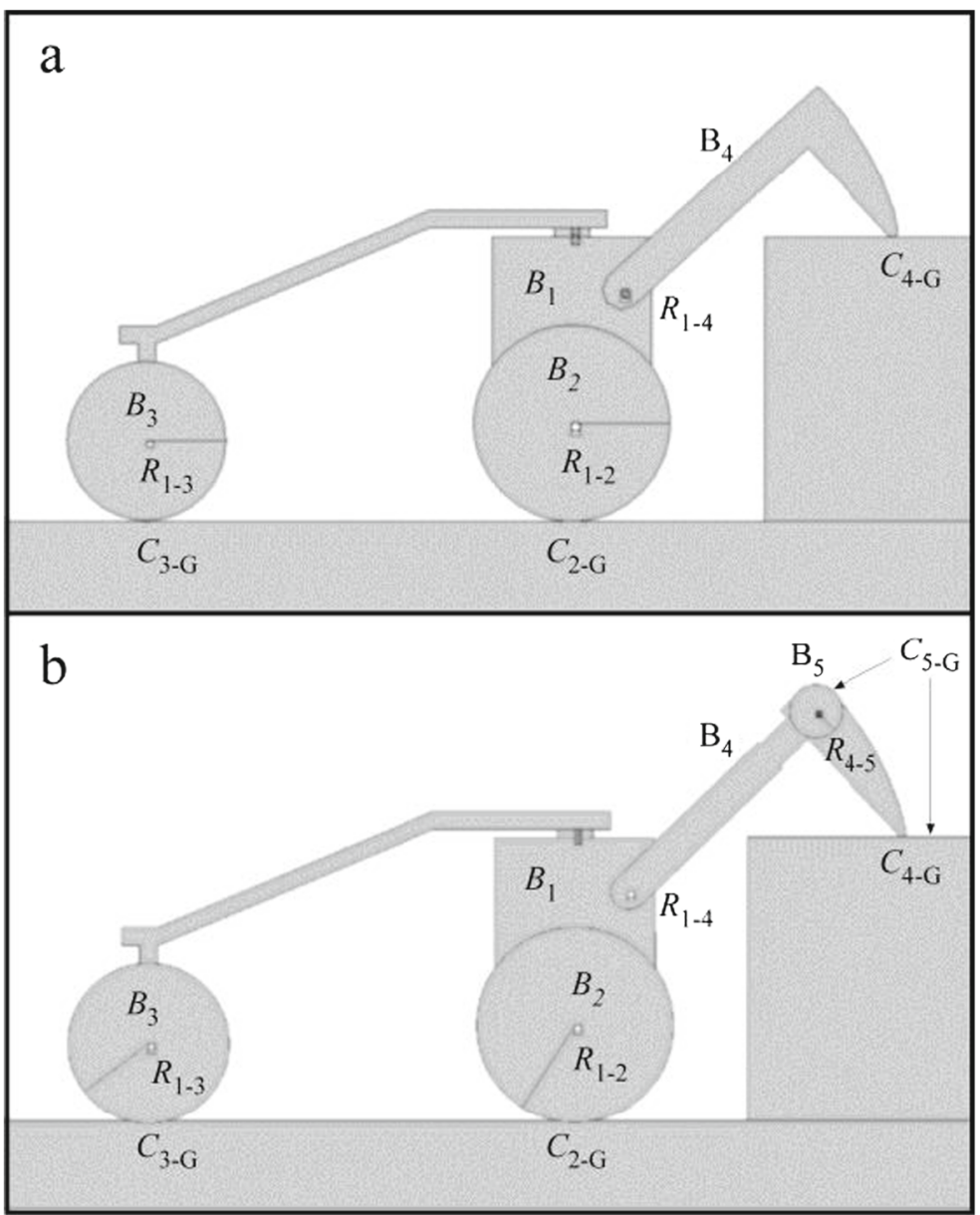

Figure 12 shows the legs velocity law for both models (red) and the one-way bearings speed, only for

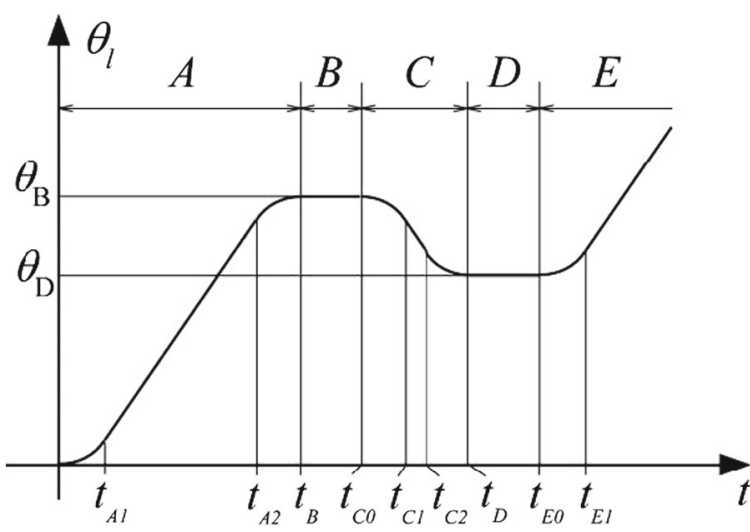

Fig. 11 Step climbing leg motion law the model equipped with auxiliary wheels. Figures 13 and 14 compare the front wheels moment: during phases $C$ and $D$, between $4.10 \mathrm{~s}$ and $4.90 \mathrm{~s}$, the rotation of the auxiliary wheels (Fig. 12) switches from sliding friction between legs and horizontal step surface to lower rolling friction, thus reducing the wheel torque, lowering the risk of slip and improving the reliability of the manoeuvre; in this time period the presence of the auxiliary wheels influences also the legs moment (Fig. 15), while the robot trajectory is nearly the same for the two models (Fig. 16).

The motion law parameters used in the previously discussed simulations have been selected for a standard step (160 mm of rise); both the simulation campaign and the experimental tests have shown that Mantis can climb higher square steps (up to about 200 $\mathrm{mm}$ of rise); while for steps lower than $160 \mathrm{~mm}$ the 


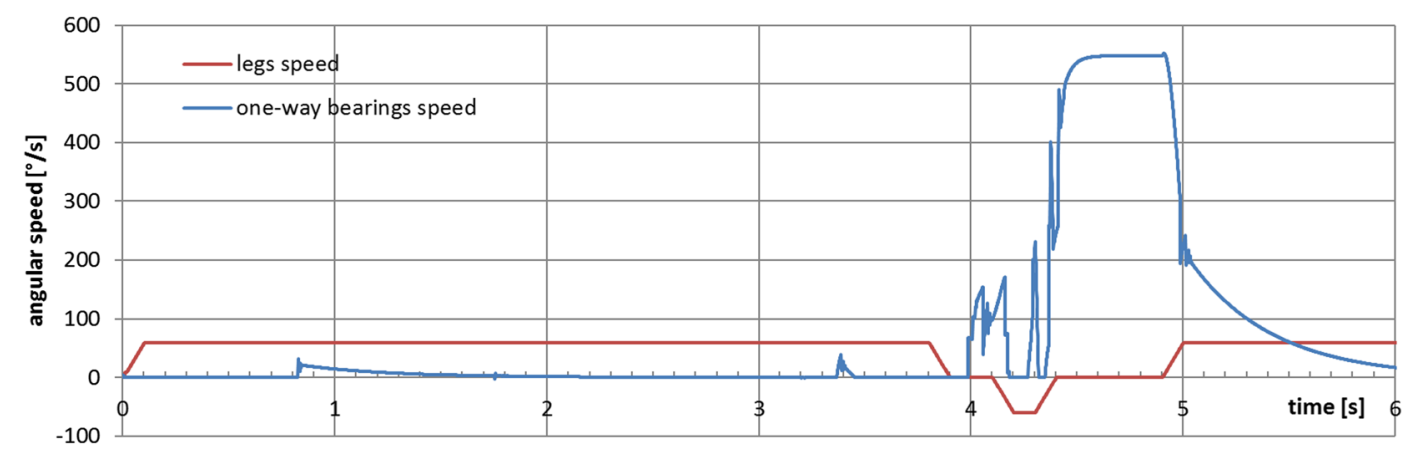

Fig. 12 Legs angular speed $(\% / \mathrm{s}$, red $)$ and oneway bearing speed $(\%$ s, blue $)$

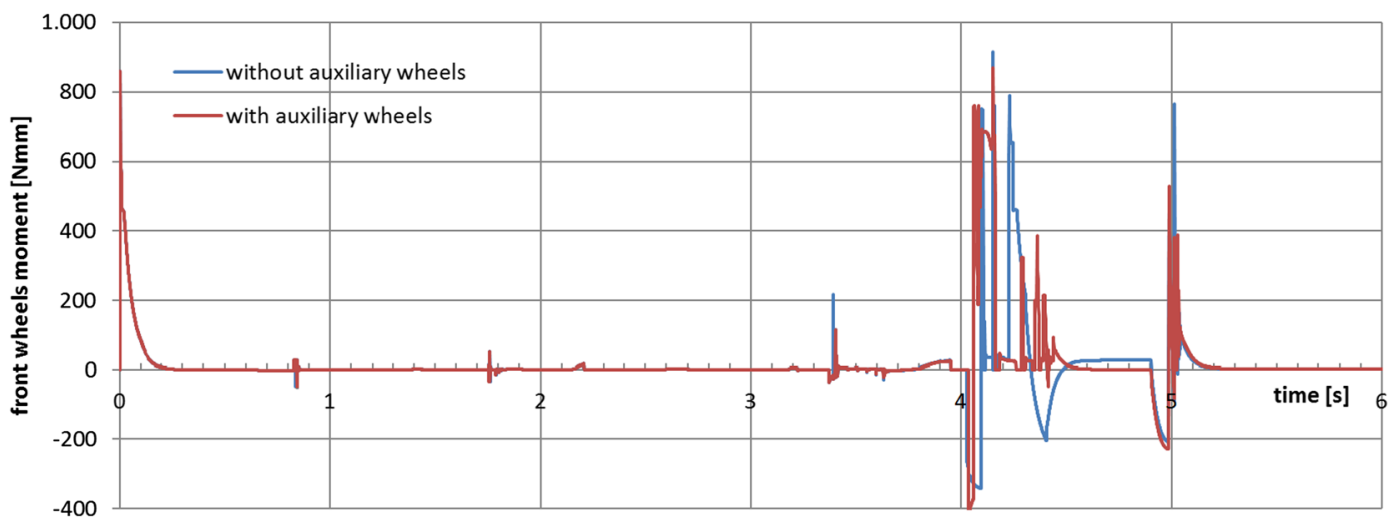

Fig. 13 Front wheels moment [Nmm] without auxiliary wheels (blue) and with auxiliary wheels (red)

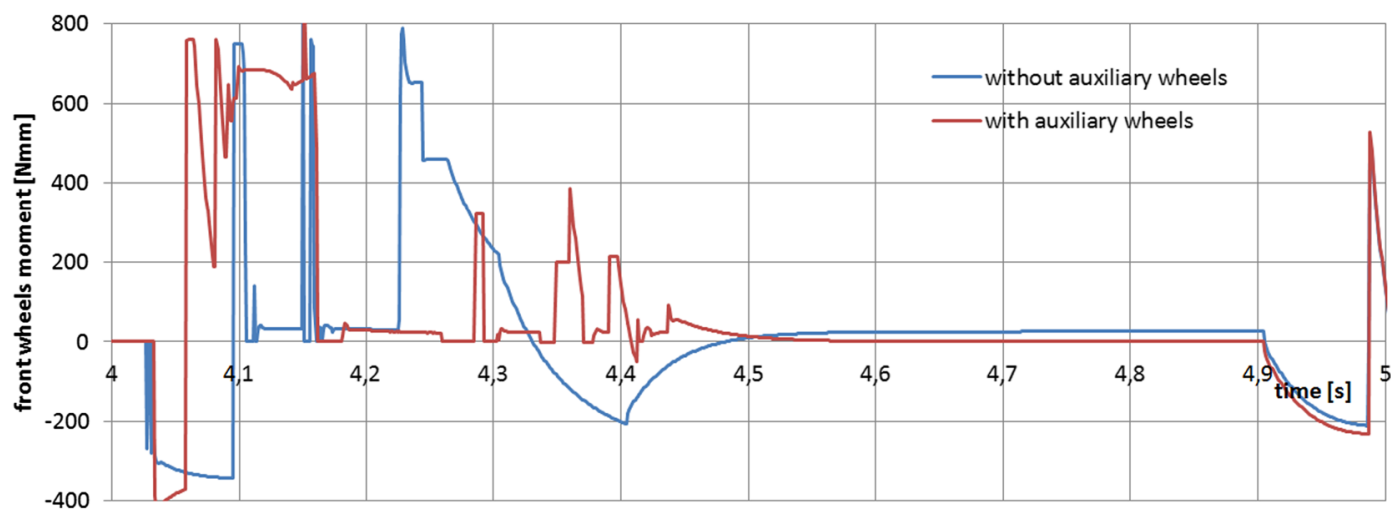

Fig. 14 Front wheels moment [Nmm]: detail of the phases C and D 


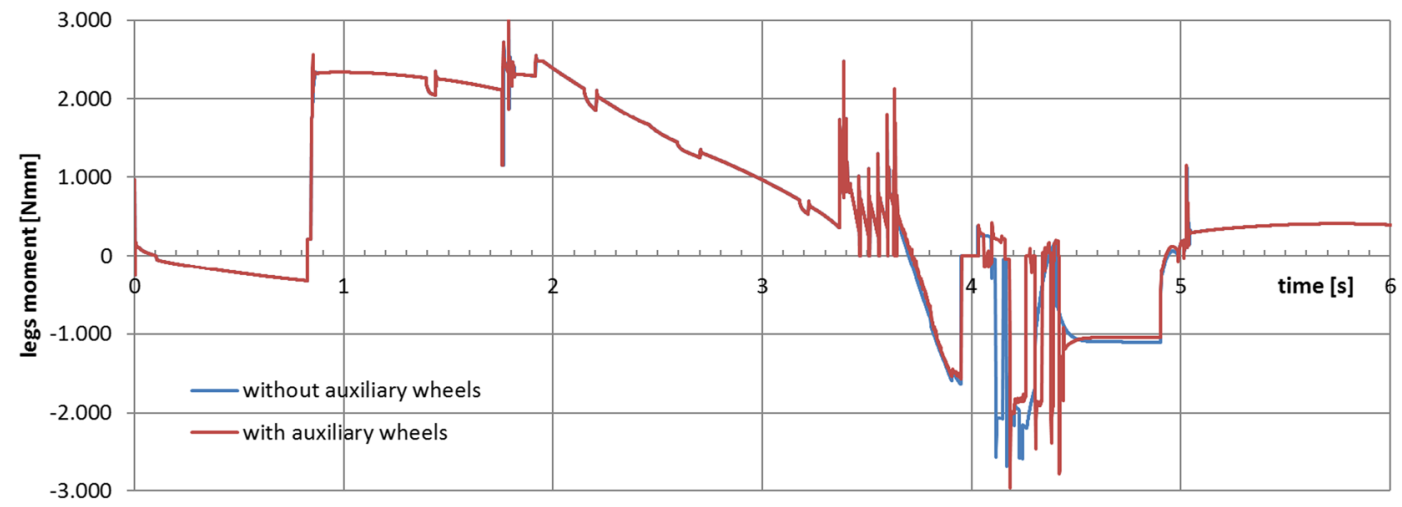

Fig. 15 Legs moment [Nmm] without auxiliary wheels (blue) and with auxiliary wheels (red)

same motion law parameters are suitable, in case of higher steps the angle $\theta_{\mathrm{B}}$ must be accurately tuned to perform correctly the rotation of the robot around the contact point $\mathrm{P}$, which is the most critical phase.

\section{Embodiment Design of Mantis 2.0 Legs with Auxiliary Wheels}

The overall mechanical architecture of Mantis 2.0 (Fig. 17) is similar to the first version, and the redesign is focused on the legs. Figure 18 shows the mechanical layout of the main body: the front wheels are actuated independently by the gearmotors $m_{1}$ and $m_{2}$, and the legs are actuated independently by $m_{3}$ and $m_{4}$. Four gear pairs $\left(g_{1} \ldots g_{4}\right)$ realize the transmission between the output shafts of the gearmotors $m_{1} \ldots m_{4}$ and the shafts $s_{1} \ldots s_{4}$ of front wheels and legs. The main body hosts also the motor drives, the remote control receiver, the wireless video transmitter and the batteries; room is available for additional environmental sensors.

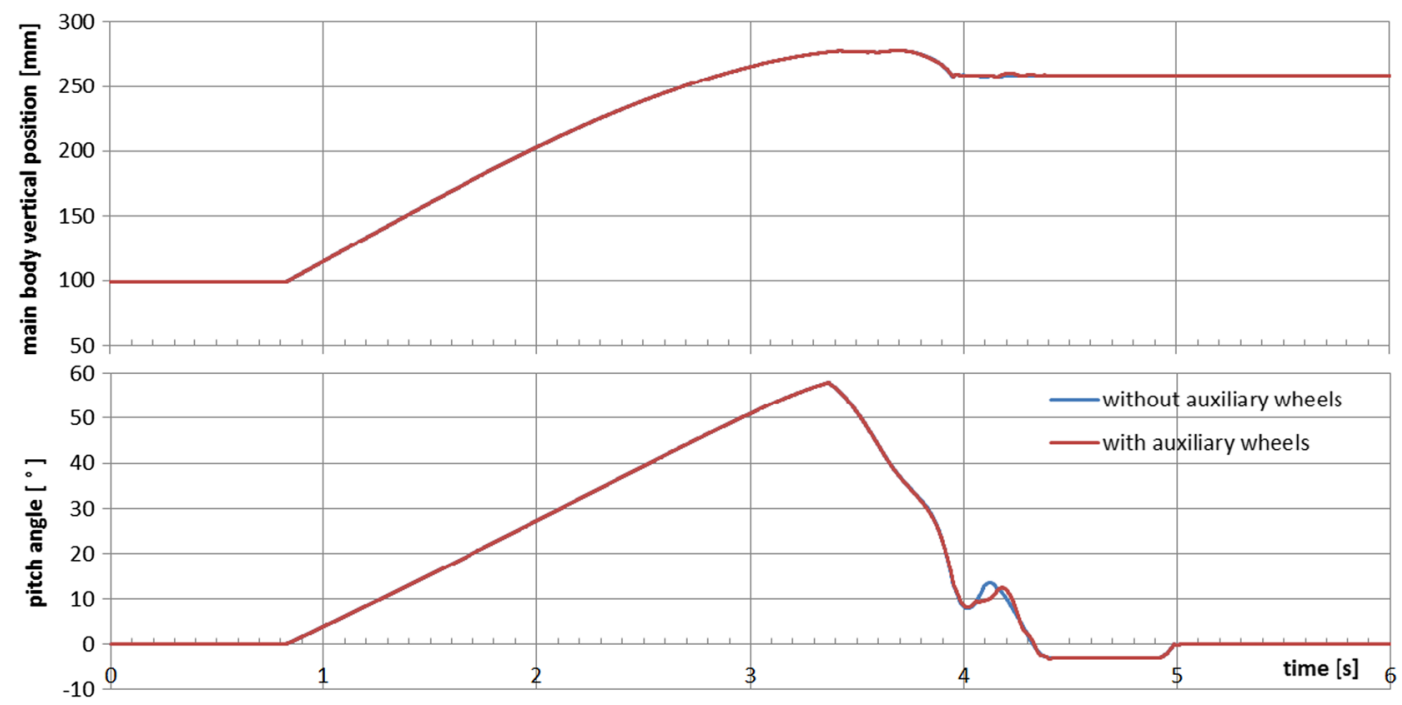

Fig. 16 Main body vertical position $[\mathrm{mm}]$ and pitch angle $\left[{ }^{\circ}\right]$ without auxiliary wheels $($ blue $)$ and with auxiliary wheels $($ red $)$ 
Fig. 17 Mantis 2.0

embodiment design: overall

view

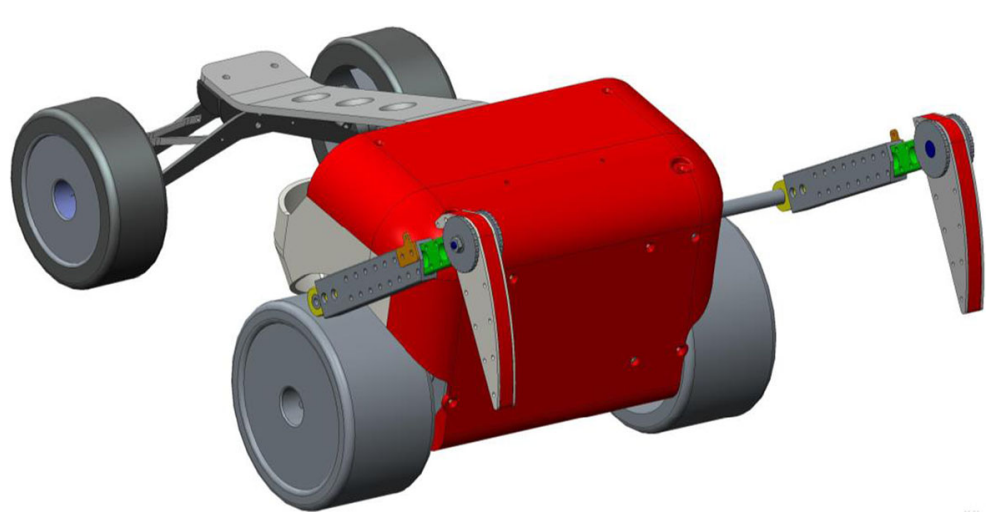

The redesign of the legs has been performed considering three main requirements:

- introduction of the auxiliary wheels to improve the reliability of the final phase of step climbing, as discussed in Sections 5 and 6;

- possibility of varying the legs length: even if the parameter $\gamma_{l}$ can be optimized in simulation for a square step, this geometrical ratio influences the robot behaviour in many other operative conditions, for example while performing mixed leg-wheel locomotion on unevenness and obstacles; therefore variable-length legs are useful for an exhaustive experimental campaign;

- realization of the elastic return force of the passive degrees of freedom of the legs end by coil springs instead of flexible plates, to have the possibility of quickly changing stiffness and preload.

The resulting leg design is shown in Figs. 19 and 20. The legs are telescopic; the elastic preload of the leg ends $a$ can be tuned by moving the plates $b$ (Fig. 20);
Fig. 18 Mantis 2.0 embodiment design: mechanical layout of the main body

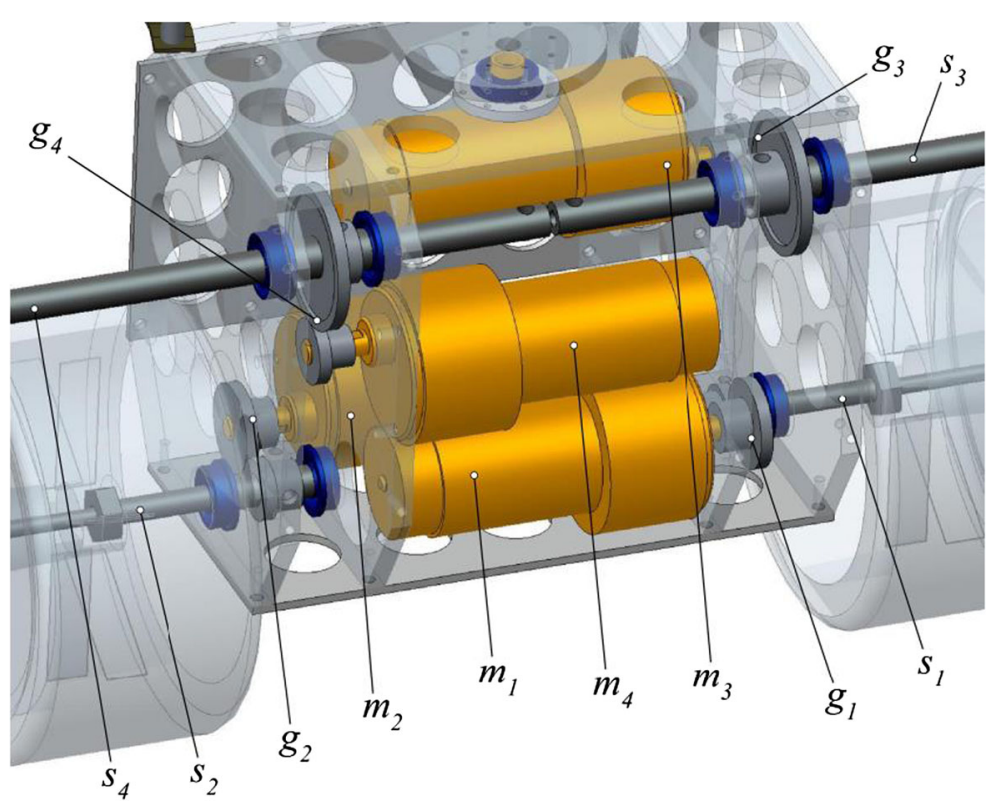



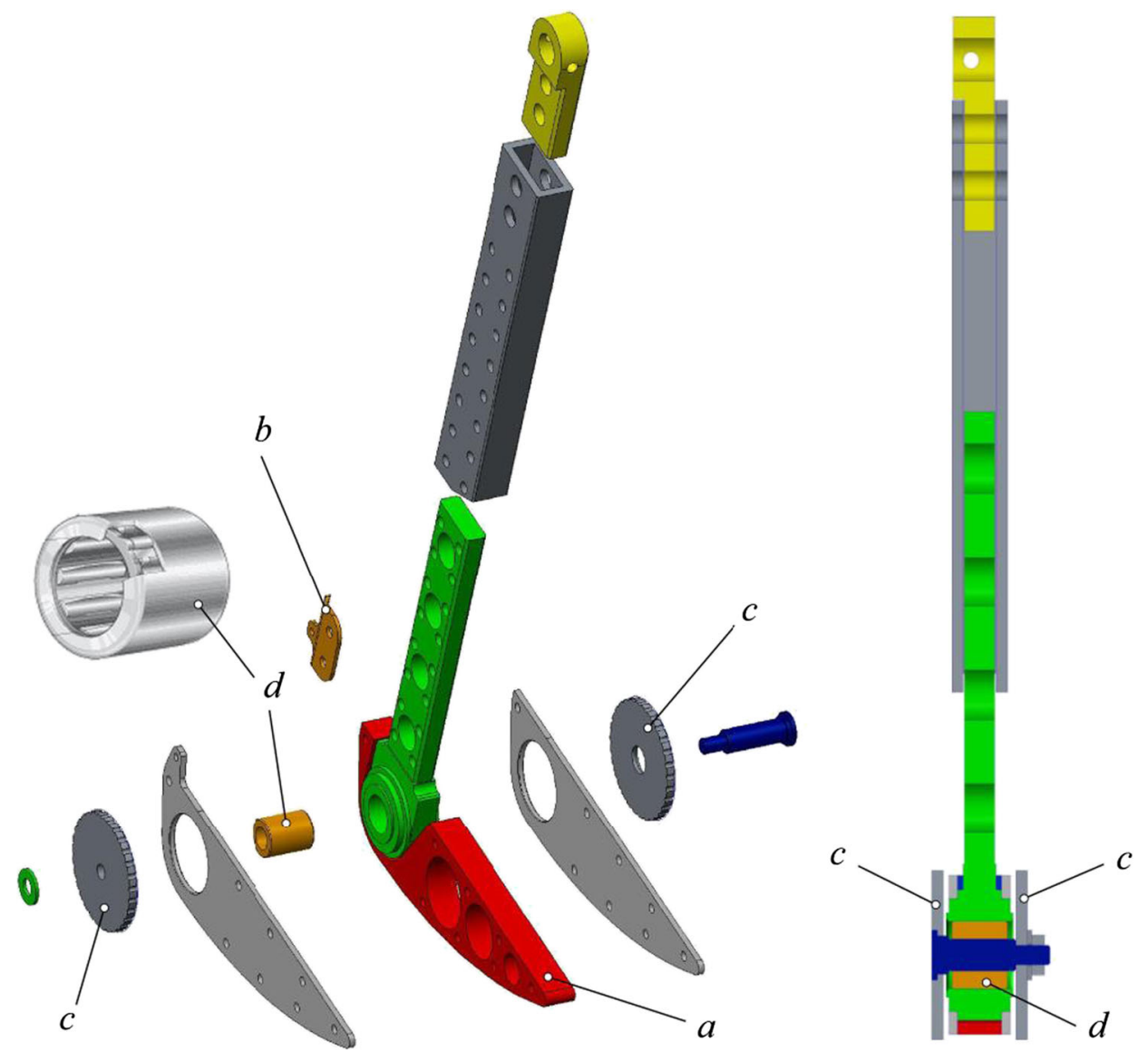

Fig. 19 Variable-length legs with auxiliary wheels: exploded view and section

Fig. 20 Variable-length legs with auxiliary wheels: working principle

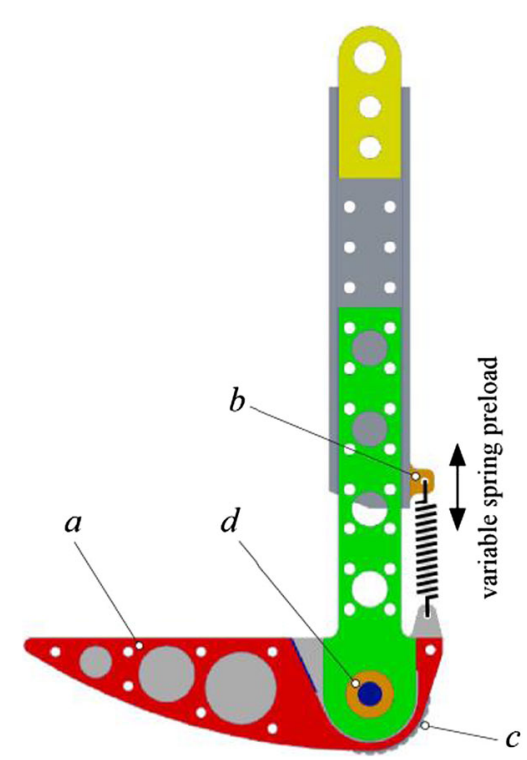

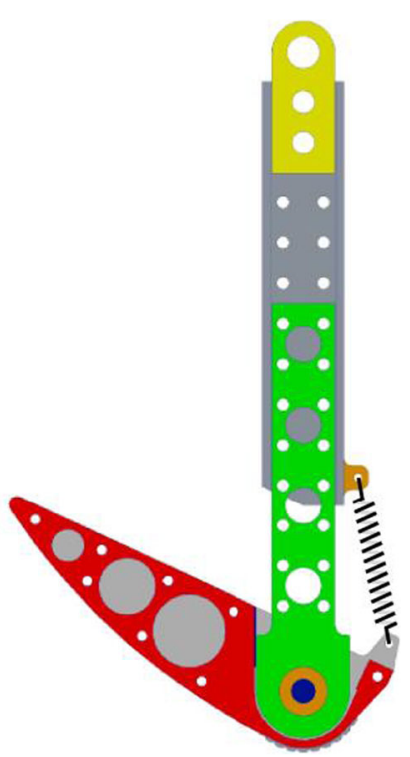


the relative motion between legs and auxiliary wheels $c$ is constrained by the one-way bearing $d$. The realization of the Mantis 2.0 prototype is in progress.

\section{Conclusions}

The experimental tests on the Mantis 1.0 prototype have shown that the adopted hybrid solution is efficient, flexible and suitable for a wide range of structured and unstructured environments, thanks to its mixed locomotion which conjugates energetic efficiency, stable camera vision on flat terrains and climbing ability. Similarly to stepping triple wheels robots, and unlike rotating legs robots, Mantis has good energetic efficiency, due to the wheeled locomotion without continuous impacts on the terrain, and stable vision. An advantage over stepping triple wheels robots is the capability of climbing square steps higher than the robot itself in rest position, thanks to the shape of the rotating legs.

During step climbing, the most critical phase is the last, when the front wheels must move forward the robot, which is suspended on the legs pointing backward to lift the rear axle. Even if in general the wheels traction overcomes the legs-step friction, the tests have highlighted that in particular conditions wheels may slip during this phase, lowering the repeatability and reliability of the step climbing manoeuvre. In order to solve this problem, the no-slip condition in the final phase of step climbing has been analysed, and a partial redesign of the legs, with the introduction of unidirectional auxiliary wheels, has been tested by means of multibody simulation.

The next step of the research is an exhaustive experimental campaign on the Mantis 2.0 prototype to confirm the effectiveness of the new leg design, not only for square step climbing but also in hybrid leg-wheel locomotion on uneven terrains and irregular obstacles.

Moreover, another important topic is the development of an automatic guidance system for step/stair climbing, capable of controlling the robot motion with a proper leg-wheel coordination; to this aim, the main issue is the design of a navigation system capable of calculating path and motion planning, with sufficient reliability and robustness to disturbances, by merging the information coming from on-board sensors and terrain mappings. In particular, the main body of the robot should be equipped with a video camera and an infrared, laser or ultrasonic distance sensor mounted on a pan/tilt support. The vision system sends the images to the control station, where they are analysed automatically by a scene recognition system or by a human operator. Once detected the presence of the step or of the stair, the step/stair profile can be reconstructed by means of the movable distance sensor [20]. Then the guidance system positions the robot in front of the step and starts the climbing sequence discussed in Section 6, tuning the motion law of legs and wheels as a function of the step rise and going. Once started the step climbing sequence, distance sensors placed on the front of the main body and under the rear axle can be used to measure the actual relative position between the robot and the step/stair profile, in order to monitor the manoeuvre progress, thus providing sufficient robustness to the guidance system. The logic of variation of the climbing motion sequence as a function of the step geometry can be synthetized by using the proposed multibody model.

\section{References}

1. Murphy, R.R.: Rescue robotics for homeland security. Communications of the ACM - Homeland security 47(3), 66-68 (2004)

2. Playter, R., Buehler, M., Raibert, M.: Bigdog. Proc. of the SPIE Defense \& Security Symposium, Unmanned Systems Technology (2006)

3. Quaglia, G., Bruzzone, L., Bozzini, G., Oderio, R., Razzoli, R.: Epi.q-TG: mobile robot for surveillance. Ind. Robot. 38(3), 282-291 (2011)

4. Birk, A., Carpin, S.: Rescue robotics: a crucial milestone on the road to autonomous systems. Adv. Robot. 20(5), 596605 (2006)

5. Snyder, R.G.: Robots assist in search and rescue efforts at WTC. IEEE Robot. Autom. Mag. 8(4), 26-28 (2001)

6. Hamel, W.R., Cress, R.C.: Elements of telerobotics necessary for waste clean up automation. Proc. IEEE Intl. Conf. on Robotics and Automation, pp 393-400 (2001)

7. Siegwart, R., Nourbakhsh, I.R.: Introduction to Autonomous Mobile Robots. MIT Press, Cambridge (2004)

8. Seeni, A., Schafer, B., Rebele, B., Tolyarenko, N.: Robot mobility concepts for extraterrestrial surface exploration. Proc. of the IEEE Aerospace Conference, pp. 1-14 (2008)

9. Bruzzone, L., Quaglia, G.: Locomotion systems for ground mobile robots in unstructured environments. Mech. Sci. 3, 49-62 (2012)

10. Altendorfer, R., Moore, N., Komsuoglu, H., Buehler, M., Brown, H.B. Jr., McMordie, D., Saranli, U., Full, R., Koditschek, D.E.: RHex: a biologically inspired hexapod runner. Auton. Robot. 11(3), 207-213 (2001) 
11. Quinn, R.D., Nelson, G.M., Bachmann, R.J., Kingsley, D.A., Offi, J.T., Allen, T.J., Ritzmann, R.E.: Parallel complementary strategies for implementing biological principles into mobile robots. Int. J. Robot. Res. 22(3),169-186 (2003)

12. Herbert, S.D., Drenner, A., Papanikolopoulos, N.: Loper: A Quadruped-hybrid stair climbing robot. Proc. 2008 IEEE Conf. on Robotics and Automation, May 19-23. Pasadena, CA (2008)

13. Siegwart, R., Lauria, M., Maeusli, P.A., Van Winnendael, M.: Design and implementation of an innovative micro rover. Proc. of Robotics 98, 3rd Conference and Exposition on Robotics in Challenging Environments (1998)

14. Quaglia, G., Bruzzone, L., Oderio, R., Razzoli, R.: Epi.Q Mobile robots family. Proc. of the ASME 2011 International Mechanical Engineering Congress \& Exposition IMECE2011, November 11-17, pp. 1165-1172. Denver, CO (2011)

15. Quaglia, G., Oderio, R., Bruzzone, L., Razzoli, R.: A modular approach for a family of ground mobile robots. Int. J. Adv. Robot. Syst. 10 (2013)

16. Bruzzone, L., Fanghella, P.: Multibody simulation of the dynamic behaviour of a hybrid leg-wheel ground mobile robot. Proc. of the 33rd IASTED International Conference on Modelling, Identification and Control MIC 2014, February 17-19, pp. 237-243. Innsbruck, Austria (2014)

17. Bruzzone, L., Fanghella, P.: Mantis hybrid leg-wheel robot: stability analysis and motion law synthesis for step climbing. Proc. of MESA 2014, 10th IEEE/ASME International Conference on Mechatronic and Embedded Systems and Applications, September 10-12. Senigallia, Italy (2014)

18. Bruzzone, L., Fanghella, P.: Mantis: hybrid leg-wheel ground mobile robot. Ind. Robot. 41(1), 26-36 (2014)

19. Garcia, E., Estremera, J., Gonzalez de Santos, P.: A classification of stability margins for walking machines. Robotica 20, 595-606 (2002)

20. Fujita, T., Kondo, Y.: 3D terrain sensing system using laser range finder with arm-type movable unit. In: Satoru, G. (ed.) Robot Arms, pp. 159-174. InTech, Rijeka (2011)
Luca Bruzzone was born in Savona in 1973. He received his Master degree, magna cum laude, in Mechanical Engineering at the University of Genoa in 1997, with a specialization in Industrial Automation and Robotics and a degree thesis on the design of a parallel robot co-operating in deburring tasks. In 1998 he joined the Material Handling division of Techint Italimpianti, working as mechanical, structural and automation designer of continuous ship unloaders and other machines. Since 1999 he has been Assistant Professor of Mechanics of Machinery at the University of Genoa. His research interest is focused on mechanics and control of robots and automation devices, and in particular: parallel robotics, mobile robotics, cooperation of robots in complex tasks with force control, robot control algorithms, design of miniaturized devices and microgrippers with flexible joints, design of mechatronic systems actuated by electrical linear motors, fractional-order control of mechatronic devices.

Pietro Fanghella Pietro Fanghella was born in Genoa in 1955. After obtaining the Master degree in Mechanical Engineering, from 1980 to 1983 he was at the CAD-CAE Division of Italimpianti, where he developed innovative software tools for computer aided mechanical engineering and plant design. In 1983 he became Assistant Professor at the University of Genoa. In 1984 he received a CNR-NATO grant as visiting scholar at the University of Illinois at Chicago, working with Professor F.L. Litvin. In 1990, he became Associate Professor and since 2000 he is appointed as Full Professor of Mechanics of Machines at the University of Genoa. Presently he is Director of the Mechanical Computer Aided Laboratory (MCAE-Lab) at the DIME Department and Coordinator of the Mechatronic and Automation Degree. His research activity is mainly oriented to the Theory of Mechanisms and Machines and to the Mechanical Design, and more specifically to computational kinematics and dynamics, mobility of mechanisms and robots, modular symbolic methods based on group properties, software simulation codes by object-oriented programming, mobile robotics, innovative education methods and tools in the field of mechanical and mechatronic engineering. 